\title{
Applicabilité du Cobra 2024 L à la détermination de la flore totale du lait cru
}

\author{
C Marin, A Guyot \\ Station laitière, CRA de Gembloux, chaussée de Namur, 24, 5030 Gembloux, Belgique
}

(Reçu le 15 septembre 1992; accepté le 5 mars 1993)

\begin{abstract}
Résumé - L'évaluation des caractéristiques analytiques de l'appareil Cobra 2024 L (Biocom, Les Ulis Cedex, France) pour la détermination en routine de la flore totale du lait cru a été réalisée dans le cadre de son agrément en Belgique. L'étude de la répétabilité a été effectuée par l'analyse en double de 168 échantillons de lait cru de la collecte locale par le Cobra et la méthode de numération de la flore aérobie mésophile selon la norme FIL 100B 1991. L'écart type moyen de répétabilité exprimé en log UFC/ml a été, pour l'appareil réglé en routine, de 0,084 correspondant à un écart type relatif géométrique moyen exprimé en pourcentage d'UFC/ml de $25 \%$. La relation entre les résultats fournis par le Cobra $2024 \mathrm{~L}$ et la méthode de référence a été traduite grâce à une régression linéaire après transformation des données en leurs logarithmes. L'équation établie à partir de 164 résultats est de $: \log ($ Réf) $=0,749 \log$ (Cobra) $+0,494$ avec un écart type résiduel de 0,306 log UFC/ml. Le classement des résultats selon les normes utilisées en Belgique pour le paiement du lait cru (0$100000 \mathrm{UFC} / \mathrm{ml}, 100000-300000 \mathrm{UFC} / \mathrm{ml}$ et $>300000 \mathrm{UFC} / \mathrm{ml}$ ) permet d'atteindre $75,2 \%$ de correspondance en classement sans l'intervention de la relation de calibrage, l'utilisation de celle-ci n'améliorant que faiblement ce résultat $(75,6 \%)$. Le pourcentage de contamination d'un échantillon sur le suivant (carry-over) est inférieur à $0,1 \%$. La teneur en cellules somatiques n'a pas d'effet important sur le classement du lait cru aux seuils des normes de 100000 et de $300000 \mathrm{UFC} / \mathrm{ml}$.
\end{abstract}

\section{Cobra / lait cru / numération bactérienne / répétabilité / classement}

Summary - Suitability of the Cobra $2024 \mathrm{~L}$ for the total bacterial count in raw milk. The evaluation of the analytical characteristics of the Cobra $2024 \mathrm{~L}$ (Biocom, Les Ulis Cedex, France) for the total bacterial count in raw milk has been established in order to obtain the official approval of the instrument in Belgium. A series of 168 farm raw milk samples were analyzed in duplicate by the Cobra $2024 \mathrm{~L}$ and by the IDF reference standard plate count method. The repeatability SD of Cobra, expressed in log CFU/ml, was 0.084 corresponding to a geometric relative SD of $25 \%$ CFU/ml. After the logarithmic transformation of the data, a linear regression was observed between the Cobra and the reference method results : $\log ($ Ref $)=0.749 \log ($ Cobra $)+0.494 ; s_{y . x}=0.306 \log$ CFU $/ \mathrm{ml}$. After classification of the raw data of Cobra and the reference method according to Belgian standards 10 $100000 \mathrm{CFU} / \mathrm{ml}, 100000-300000 \mathrm{CFU} / \mathrm{ml},>300000 \mathrm{CFU} / \mathrm{ml}$ ), it was observed that $75.2 \%$ of the results fell into the same categories. This figure was not improved after calibration (75.6\%). The car- 
ry-over effect of the Cobra $2024 \mathrm{~L}$ was less than $0.1 \%$. The somatic cell count had no practical influence on the classification of raw milk according to the threshold values of 100000 and $300000 \mathrm{CFU} /$ $\mathrm{ml}$.

Cobra / raw milk / bacterial count / repeatability / classification

\section{INTRODUCTION}

Le comptage des germes totaux est une détermination analytique de base pour l'estimation de la qualité bactériologique des laits crus. La méthode de référence (FIL 100B 1991) présente de grands inconvénients qui interdisent une application normale en routine; son automatisation est difficile et le délai de réponse est trop long. Les laboratoires belges analysant la qualité du lait cru en vue du paiement ont utilisé jusqu'à présent des versions simplifiées et plus ou moins mécanisées (Thompson, Roll-tube) de la méthode de référence, de même que le Bactoscan de Foss Electric (Danemark), appareil qui compte les impulsions données par les bactéries colorées par l'acridine orange défilant en continu sur un disque rotatif.

Récemment, l'organisme interprofessionnel du Hainaut a acquis un Cobra $2024 \mathrm{~L}$, appareil basé également sur la détection des bactéries en épifluorescence. Afin de pouvoir être appliqué à la détermination du nombre de germes totaux et au paiement du lait cru à la qualité selon ce critère, il a dû être établi que le Cobra donnait des résultats équivalents à la méthode de référence. Une étude a donc été développée pour établir la relation entre les résultats du Cobra et les résultats de la méthode de référence sur des échantillons de lait cru récoltés dans le cadre de l'organisation du système belge pour le paiement du lait selon la qualité. C'est cette étude et ses résultats qui sont décrits ici.

\section{MATÉRIEL ET MÉTHODES}

\section{Le Cobra 2024 L}

Le principe de base qui a présidé à la mise au point de la série des appareils Cobra (COmptage Bactérien RApide) est l'automatisation de la méthode DEFT (Direct Epifluorescent Filter Technique) (Pettipher et al, 1980). La technique DEFT originelle présentait comme inconvénient pour l'analyse en routine, une inadaptation aux cadences élevées et un coût relativement élevé. L'automatisation réalisée avec l'instrumentation Cobra remédie à cet inconvénient. La société Biocom (24 avenue de la Baltique, BP 53, 91942, Les Ulis Cedex, France) a développé plusieurs configurations d'appareils Cobra selon les applications et les cadences d'analyses souhaitées. Pour le domaine laitier, les applications principales sont le dénombrement des germes totaux et celui des cellules somatiques dans le lait cru (Bisconte, 1991).

Le Cobra est un système instrumental modulaire. La configuration Cobra $2024 \mathrm{~L}$ comprend trois modules, un module de distribution et de préparation des échantillons, un module de fittration et de coloration des bactéries (ou des cellules) et un module d'analyse microscopique et de traitement des données.

\section{Module de distribution et de préparation des échantillons}

Ce module est formé d'un appareil automatisé de prélèvement à l'aide d'une seringue. II reçoit un bloc compact interchangeable en polypropylène creusé de 24 puits (4 rangées de 6 puits). Le bloc peut être soumis à des températures d'incubation correspondant aux températures d'activités optimales d'enzymes intervenant dans la préparation des échantillons. Le distributeur $X Y$ procède de manière séquentielle pour alimenter les 24 puits. 


\section{Module de filtration et de coloration}

Cette unité est constituée d'un automate programmable intégrant plusieurs pompes. Un tiroir supporte le filtre d'analyse monté dans un cadre rigide et le bloc de polypropylène avec ses 24 puits contenant les échantillons préparés.

\section{Module d'analyse et de traitement des données}

Ce module comprend un microscope à épifluorescence avec caméra et écran vidéo, une platine motorisée $X Y Z$, une unité de fluorescence, un analyseur d'images avec micro-ordinateur. Une pompe à vide aspire et aplatit le filtre sur la platine pour qu'il présente une surface parfaitement plane. Un système de focalisation automatique permet des ajustements très fins (Bisconte, 1990). Le facteur de multiplication est de 20440 (résultat final exprimé en UFC/ml).

La technique Cobra a été conçue avec le souci de respecter l'environnement. En effet, elle minimise les rejets de réactifs dans celui-ci et emploie des accessoires réutilisables. Le traitement des échantillons s'effectue en système fermé et les vapeurs éventuelles sont filtrées avant leur libération dans l'air ambiant. Les réactifs utilisés par échantillon sont en quantité excessivement faible, de l'ordre du $\mu$ l. Cela limite les problèmes de stockage et d'élimination des déchets.

\section{Échantillons}

Les échantillons de lait cru ont été prélevés à la ferme par l'échantillonneur mécanique du camion de collecte pendant I'hiver 1991-1992. Ils ont été maintenus dans l'eau glacée $\left(<2^{\circ} \mathrm{C}\right)$ jusqu'à l'arrivée au laboratoire de l'organisme interprofessionnel de Soignies, où les déterminations ont débuté dès le lendemain matin (après 24 à $30 \mathrm{~h}$ ).

\section{Méthode de référence pour la détermination des germes totaux}

La méthode FIL 100 B (1991) a été exactement suivie. La troisième dilution décimale de chaque échantillon est ensemencée en double. Après incubation pendant $3 \mathrm{j}$ à $30^{\circ} \mathrm{C}$, les comptages sont effectués visuellement. Le résultat considèré est la moyenne des 2 doubles.

\section{Méthode simplifiée pour la détermination des germes totaux (Thompson)}

Un $\mu l$ de lait de chaque échantillon est prélevé et distribué mécaniquement avec de l'eau de Ringer et le milieu de culture dans des boîtes de Pétri. Ces boîtes sont incubées 3 j à $30^{\circ} \mathrm{C}$ et lues par un compteur automatique. Cette méthode est beaucoup plus rapide et moins fastidieuse à mettre en cuvre que la méthode de référence.

\section{Méthode Cobra}

Le protocole d'analyse et les réactifs sont décrits dans le Guide utilisateur Biocom. Les blocs en polypropylène chauffés à $50^{\circ} \mathrm{C}$ sont additionnés de $300 \mu \mathrm{l}$ d'eau stérile par puits. Le distributeur automatique prélève et distribue $100 \mu \mathrm{l}$ des échantillons de lait, puis un mélange de $800 \mu \mathrm{l}$ de triton 0,5\% - trypsine, Biocom (670-130). Le bloc est laissé à la température du laboratoire pendant $10 \mathrm{~min}$, et il est transmis dans le module de filtration et de coloration. La pression de filtration est de 2 bars et le temps de filtration minimum est de $6 \mathrm{~s}$. Les 24 impacts de filtration sont soumis au rinçage par $300 \mu l$ d'un tampon citrate de $\mathrm{pH}=3$, puis sont colorés à l'acridine orange par $200 \mu \mathrm{l}$ d'une solution à $0,025 \%$ de $\mathrm{pH}=4$ (temps de coloration de $3 \mathrm{~min}$ ). Le filtre est de nouveau rincé par le tampon citrate de $\mathrm{pH}=3(750 \mu \mathrm{l})$ et soumis à la filtration sous pression, puis séché pendant $1 \mathrm{~min}$. Le badge avec les 24 échantillons est déposé et fixé sur le poste de lecture et d'analyse. Il est à remarquer que le prélèvement des échantillons ne nécessite pas un préchauffage et que l'incubation des blocs pendant 10 min pour permettre au mélange triton-trypsine de solubiliser les cellules et les globules gras a été effectuée sans replacer les blocs à $50^{\circ} \mathrm{C}$.

Le Cobra $2024 \mathrm{~L}$ a été conçu pour effectuer de 300 à 1000 déterminations de germes dans le lait par jour. En pratique, le premier badge est 
lu $30 \mathrm{~min}$ après le début de la distribution des 24 échantillons. Pour un échantillon de lait, le nombre de champs d'analyse est inversemment proportionnel à sa charge bactérienne. Par exemple, pour un lait de 30000 à 70000 germes $/ \mathrm{ml}$, le logiciel prend en compte un maximum de 15 champs mais pour un lait $>300000$ germes $/ \mathrm{ml}$ il considère seulement 5 champs. Selon la précision souhaitée (maximale dans notre cas à 100000 germes $/ \mathrm{ml}$ ), l'ordinateur gère automatiquement les mesures et interrompt le comptage lorsque la moyenne cumulée des lectures (entre 5 et 15 a Soignies) se stabilise. Dans ces conditions, le seuil de détection est de l'ordre de 20000 germes/ml (Grappin. 1990).

Cela a permis à un seul opérateur de déterminer 144 échantillons $(6 \times 24)$ par heure. Cette cadence était largement suffisante pour les besoins de l'organisme interprofessionnel (environ 450 échantillons/j), mais pourrait être augmentée en affectant une personne ou un module supplémentaire aux tâches de préparation. La mesure de champs supplémentaires est possible selon les besoins. Elle devrait augmenter la répétabilité en diminuant cependant la cadence d'analyse.

\section{Détermination des cellules somatiques}

Le nombre de cellules somatiques a été déterminé par le Fossomatic 250 (Foss Electric) en service au laboratoire de Soignies. La concordance des résultats Fossomatic avec ceux de la méthode de référence est vérifiée régulièrement par l'introduction d'échantillons étalons de Cecalait (Poligny, France) et d'analyses circulaires interlaboratoires belges.

\section{Évaluation du Cobra}

\section{Répétabilité}

Le Cobra du laboratoire de Soignies étant l'unique appareil de ce type à notre disposition, parmi les paramètres exprimant la fidélité, la répétabilité seule a été déterminée. La répétabilité $r$ définie par la norme FIL 128 (1985) est la valeur au-dessous de laquelle est située avec une probabilité spécifiée, la valeur de la différence entre 2 résultats individuels obtenus dans les mêmes conditions. Le niveau de probabilité choisi étant de 0,95 , la répétabilité est l'écart arithmétique entre 2 déterminations ayant une probabilité de $95 \%$ de ne pas ètre dépassée (Grappin, 1976). La répétabilité, calculée à partir des données transformées en unités logarithmiques, est exprimée à partir de l'écart type de répétabilité $s_{r}$ par la formule

$$
r=2,83 \cdot \mathrm{s}_{\mathrm{r}}\left(\mathrm{s}_{\mathrm{r}}=\left(\sum \mathrm{di}^{2} / 2 n\right)^{1 / 2}\right.
$$

où $\mathrm{di}=$ différence entre 2 doubles et $n=$ nombre d'échantillons mesurés par la méthode.

L'écart type relatif géométrique de répétabilité GRSD (Dasen et al, 1991, Piton et Grappin, 1991) en données non transformées a été calculé selon la formule suivante:

$$
\text { GRSD }\left(\text { en UFC/ml) }=\left(10^{S_{r}}-1\right) \times 100\right.
$$

Le GRSD a été calculé pour l'ensemble des résultats et pour 4 intervalles de teneur en germes $(0$ à 50000,51000 à 100000,101000 à $300000,>300000 \mathrm{UFC} / \mathrm{ml})$.

La répétabilité du Cobra $2024 \mathrm{~L}$ a été calculée sur un lot de 168 échantillons de lait cru analysés en double. Á titre de comparaison, la répétabilité de la méthode de référence a été effectuée sur un lot de 35 échantilions de lait cru.

\section{Relation entre résultats Cobra et résultats Référence (exactitude)}

Les résultats obtenus par le Cobra sont exprimés en UFC/ml, comme la méthode de référence. Avec les paramètres techniques correctement réglés, notamment les paramètres de contraste, de luminosité et le seuil de détection, la mesure a donné des résultats en moyenne égaux à ceux de la méthode de référence, sans calibrage. L'étude de la relation entre les résultats "référence" comme variable dépendante et les résultats Cobra comme variable indépendante permettra de se rendre compte du bon réglage des paramètres et de la nécessité du calibrage.

On a d'abord transformé les lectures UFC/ml en leurs logarithmes afin de rapprocher la distribution des résultats d'une distribution normale. Nous avons ensuite choisi d'appliquer une régression linéaire après avoir observé qu'une ré- 
gression quadratique ne rendait pas mieux compte de la dispersion des résultats. Un classement prédictif des résultats a été réalisé selon les seuils officiels utilisés en Belgique pour l'attribution des points de pénalisation, soit 100000 et $300000 \mathrm{germes} / \mathrm{ml}$. Le classement a été effectué d'une part, en utilisant directement les mesures Cobra en log UFC/ml et, d'autre part, en appliquant l'équation de régression aux mesures Cobra pour obtenir une estimation de la vraie valeur censée ètre donnée par la méthode de référence. La comparaison des classements prédictifs à l'aide des résultats bruts et des résultats estimés permettra de juger de l'opportunité d'utiliser l'équation de régression (Pettipher et al, 1992). Cette étude a été effectuée sur un lot de 165 échantillons de lait cru.

\section{Influence de la contamination d'un échantilion sur le suivant (carry-over effect)}

L'effet de la contamination d'un échantillon sur le suivant a été étudié en soumettant à la détermination alternativement 12 séquences de lait chargé en micro-organismes $\left(4 \cdot 10^{5}\right.$ à $\left.2.106 / \mathrm{ml}\right)$ et d'eau stérile.

\section{Influence du nombre de cellules somatiques}

L'influence de la teneur en cellules somatiques sur le comptage des germes a été étudiée grâce à une équation de régression multiple du type

$$
y=a+b x+c z
$$

où $y=\log$ (Thompson), $x=\log$ (Cobra) et $z=$ $\log$ (Cellules).

La méthode de comparaison a été la méthode Thompson avec lecture Biomatic, qui a permis de disposer de davantage de résultats que la méthode de référence en raison de sa mécanisation. Cette relation a été établie sur un lot de 503 échantillons de lait cru.

\section{RÉSULTATS}

\section{Répétabilité}

La répétabilité du Cobra a été calculée à partir des résultats de 168 échantillons de lait cru (de moyenne égale à 84000 UFC/ $\mathrm{ml}$ avec une amplitude de 4000 à 867000 $\mathrm{UFC} / \mathrm{ml}$ ) analysés en double, en plaçant les doubles côte à côte sur le filtre d'analyse. L'écart type de répétabilité calculé sur les données transformées (selon la formule décrite précédemment) a été de 0,084 , la répétabilité de 0,238 correspondant à un GRSD de $21,34 \%$.

La répétabilité de la technique Cobra est liée au nombre de bactéries présentes dans l'échantillon. Les écarts types de répétabilité et les GRSD de la méthode Cobra ont été calculés en fonction du nombre de germes, en considérant 4 catégories comme indiqué dans le tableau $\mathrm{I}$.

La répétabilité de la numération des germes par la méthode Cobra est meilleure avec l'augmentation du nombre de germes. La répétabilité de la méthode de référence a été déterminée en même temps à partir de 35 échantillons de lait cru (de moyenne égale à $132000 \mathrm{UFC} / \mathrm{ml}$ avec une amplitude de 5000 à 443000 $\mathrm{UFC} / \mathrm{ml}$ ) avec une répétition complète de tout le processus. Nous avons observé un écart type de répétabilité de 0,128 , une répétabilité de 0,362 et un GRSD de $34,3 \%$. Ces valeurs sont de l'ordre de celles annoncées dans la littérature (Grappin et al, 1985; Suhren et al, 1992).

La répétabilité du Cobra 2024 L est, dans ces conditions, au moins aussi bonne que celle de la méthode de référence. A noter, le calibrage du Cobra améliore en- 
Tableau I. Répétabilité du Cobra 2024 L.

Repeatability of Cobra 2024 L.

\begin{tabular}{lllr}
\hline Catégories & $s_{r}$ & $\begin{array}{l}\text { GRSD } \\
(\%)\end{array}$ & $N$ \\
\hline 0 à 50 000 UFC/ml & 0,097 & 25,03 & 85 \\
50000 à 100 000 UFC/ml & 0,078 & 19,67 & 36 \\
100000 à 300 000 UFC/ml & 0,061 & 15,08 & 30 \\
$>300$ 000 UFC/ml & 0,072 & 18,03 & 17 \\
Ensemble des données & 0,084 & 21,34 & 168 \\
\hline
\end{tabular}

$\mathrm{s}_{\mathrm{r}}$ : écart type de répétabilité du Cobra en $\log$ UFC/ml; GRSD : écart type relatif géométrique de répétabilité en \% d'UFC/ml; $\mathbf{N}$ : nombre d'échantillons.

$s_{r}$ : Repeatability SD of Cobra in $\log$ CFU/ml; GRSD: geometric relative SD of repeatability in \% CFU/ml; $\mathrm{N}:$ numbers of samples.

core les paramètres de répétabilité de l'appareil avec respectivement un écart type de répétabilité de 0,063 , une répétabilité $r$ de 0,178 et un GRSD de $15,6 \%$.

\section{Relation entre résultats Cobra et résultats de la méthode de référence (exactitude)}

La figure 1 présente le diagramme de dispersion des résultats obtenus par le Cobra et par la méthode de référence.

La relation entre les 2 méthodes est définie par l'équation de régression linéaire calculée à partir des résultats obtenus sur 164 échantillons de lait cru (élimination d'une donnée aberrante du lot des 165 laits sur la base du résidu standardisé supérieur à 2,5 fois l'écart type) :

$$
\log (\text { Réf })=0,749 \log (\text { Cobra })+0,494
$$

Le coefficient de détermination $\mathrm{R}^{2}$ est de 0,571 . L'écart type résiduel $s_{y . x}$ est de 0,306 . Les moyennes des résultats de la méthode Cobra et de la méthode de réfé- rence sont très proches, et égales respectivement à 4,797 et 4,768 (en unités log). L'exécution d'un test d'égalité des moyennes (au niveau $\alpha=0,05$ ) montre cependant une différence statistiquement significative. Les résultats ont été classés

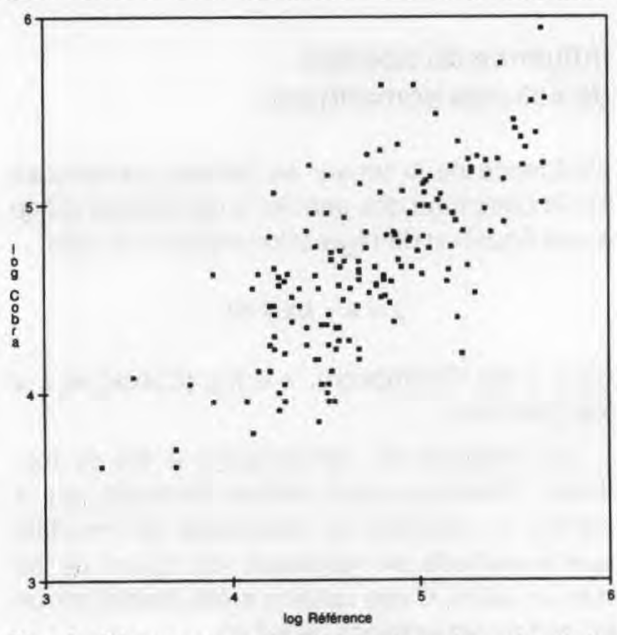

Fig 1. Diagramme de dispersion des résultats obtenus avec la méthode de référence et le Cobra $2024 \mathrm{~L}$ en log UFC/ml.

Scatter diagram of results obtained with the reference method and the Cobra 2024 L in log CFU/ $m l$. 
dans les 3 catégories établies par rapport à la méthode de référence (catégorie 1 : de 0 à $100000 \mathrm{UFC} / \mathrm{ml}$, catégorie 2 : de 101000 à $300000 \mathrm{UFC} / \mathrm{ml}$, catégorie 3 : $>300000 \mathrm{UFC} / \mathrm{ml}$ ).

Un classement effectué en utilisant les lectures directes Cobra sans application de la régression est donné dans le tableau II (165 données). Dans ce cas, $75,2 \%$ des résultats sont classés de manière similaire par le Cobra et la méthode par dilutions; $16,3 \%$ des résultats sont classés dans une moins bonne catégorie par le Cobra et $8,5 \%$ des résultats sont classés dans une meilleure catégorie par le Cobra.

Un deuxième classement est établi en fonction des valeurs Cobra estimées par la droite de régression et est donné dans le tableau III. Dans ce cas, $75,6 \%$ des échantillons sont classés de manière similaire par le Cobra et par la méthode par dilutions; $7,3 \%$ des résultats sont classés dans une moins bonne catégorie par le Cobra et $17,1 \%$ des résultats sont classés dans une meilleure catégorie par le Cobra. L'utilisation de la droite de régression n'améliore pas significativement le pourcentage de bons classements et réduit même ce pourcentage pour la troisième catégorie (tableau IV).

\section{Relation entre résultats Cobra et résultats Thompson}

La méthode Thompson a tendance à sousestimer de manière globale le nombre de germes par rapport à la méthode de référence; la relation établie antérieurement sur 330 échantillons entre les résultats Thompson et les résultats Référence est :

$$
\log (\text { Réf })=1,009 \log (\text { Thomp })+0,177
$$

Tableau II. Comparaison des classements Cobra par rapport aux classements donnés par la méthode de référence sans l'intervention de l'équation de régression.

Comparison of the grading obtained with Cobra and with the reference method without transformation by the regression equation.

\begin{tabular}{|c|c|c|c|c|c|c|}
\hline \multirow[t]{2}{*}{ Catégorie ${ }^{a}$} & \multirow[t]{2}{*}{$N$} & \multirow[t]{2}{*}{ Bien classés ${ }^{b}$} & \multirow[t]{2}{*}{$\%$} & \multicolumn{3}{|c|}{$\begin{array}{c}\text { Échantillons mal classés } \\
\text { par le Cobra }\end{array}$} \\
\hline & & & & Note $c$ & $N^{d}$ & $\%$ \\
\hline 1 & 122 & 103 & 84,4 & $1 \rightarrow 2$ & 19 & 15,6 \\
\hline 2 & 34 & 16 & 47,1 & $\begin{array}{l}2 \rightarrow 1 \\
2 \rightarrow 3\end{array}$ & $\begin{array}{r}10 \\
8\end{array}$ & $\begin{array}{l}29,4 \\
23,5\end{array}$ \\
\hline \multirow[t]{2}{*}{3} & 9 & 5 & 55,6 & $\begin{array}{l}3 \rightarrow 1 \\
3 \rightarrow 2\end{array}$ & $\begin{array}{l}1 \\
3\end{array}$ & $\begin{array}{l}11,1 \\
33,3\end{array}$ \\
\hline & 165 & 124 & 75,2 & & & \\
\hline
\end{tabular}

a Catégorie 1 : de 0 à 100000 UFC/ml; catégorie 2: de 101000 à 300000 UFC/ml; catégorie $3:>300000$ UFC/ $\mathrm{ml} ; \mathrm{N}$ : nombre d'échantillons. ${ }^{\mathrm{b}}$ Nombre d'échantillons classés correctement par le Cobra. ${ }^{\mathrm{C}}$ Changement de catégorie (Référence $\rightarrow$ Cobra). ${ }^{d}$ Nombre d'échantillons ayant changé de catégorie.

a 1st class: $0 \leq 100000$ CFU/ml; 2nd class: $101000 \leq 300000$ CFU/ml; 3rd class: $>300000$ CFU/ml; N : No of samples; ${ }^{b}$ No of samples classified by Cobra in accordance with the reference method; ${ }^{c}$ Transfer from one class to another (Reference $\rightarrow$ Cobra); ${ }^{d}$ No of samples changing class. 
Tableau III. Comparaison des classements Cobra par rapport aux classements donnés par la méthode de référence avec l'intervention de l'équation de régression.

Comparison of the grading obtained with Cobra and with the reference method with transformation by the regression equation.

\begin{tabular}{|c|c|c|c|c|c|c|}
\hline \multirow[t]{2}{*}{ Catégorie a } & \multirow[t]{2}{*}{$N$} & \multirow[t]{2}{*}{ Bien classés $b$} & \multirow[t]{2}{*}{$\%$} & \multicolumn{3}{|c|}{$\begin{array}{c}\text { Échantillons mal classés } \\
\text { par le cobra }\end{array}$} \\
\hline & & & & Note $c$ & $N^{d}$ & $\%$ \\
\hline 1 & 114 & 103 & 90,4 & $1 \rightarrow 2$ & 11 & 9,6 \\
\hline 2 & 37 & 18 & 48,6 & $\begin{array}{l}2 \rightarrow 1 \\
2 \rightarrow 3\end{array}$ & $\begin{array}{r}18 \\
1\end{array}$ & $\begin{array}{r}48,6 \\
2,7\end{array}$ \\
\hline 3 & 13 & 3 & 23,1 & $\begin{array}{l}3 \rightarrow 1 \\
3 \rightarrow 2\end{array}$ & $\begin{array}{l}1 \\
9\end{array}$ & $\begin{array}{r}7,7 \\
69,2\end{array}$ \\
\hline & 164 & 124 & 75,6 & & & \\
\hline
\end{tabular}

a Catégorie 1: de 0 à 100000 UFC/ml; catégorie 2: de 101000 à 300000 UFC/ml; catégorie $3:>300000$ UFC/ $\mathrm{ml} ; \mathrm{N}$ : nombre d'échantillons. ${ }^{\mathrm{b}}$ Nombre d'échantillons classés correctement par le Cobra. ${ }^{\mathrm{C}}$ Changement de catégorie (Référence $\rightarrow$ Cobra). ${ }^{d}$ Nombre d'échantillons ayant changé de catégorie.

a 1st class: $0 \leq 100000 \mathrm{CFU} / \mathrm{ml}$; 2nd class: $101000 \leq 300000 \mathrm{CFU} / \mathrm{ml} ; 3 \mathrm{rd}$ class: $>300000 \mathrm{CFU} / \mathrm{ml} ; \mathrm{N}$ : No of samples; ${ }^{b}$ No of samples classified by Cobra in accordance with the reference method; $c$ Transfer from one class to another (Reference $\rightarrow$ Cobra); ${ }^{d}$ No of samples changing class.

II a été établi dans cette étude à partir de 165 données la relation:

$$
\log (\text { Thomp })=0,79 \log (\text { Cobra })-0,04
$$

avec un $R^{2}=0,542$ et $s_{y \cdot x}=0,38$, impliquant une dispersion plus grande autour de la droite de régression si on considère la méthode Thompson comme la méthode de référence. Dans ce cas, une relation de calibration entre les méthodes Cobra et Thompson se révèle nécessaire. De plus, les moyennes des résultats Cobra et Thompson soit respectivement 4,797 et 4,321 , sont du point de vue statistique significativement différentes. En appliquant la relation de régression linéaire précitée, la méthode Cobra classe $88,8 \%$ des échantillons de la même manière que la méthode Thompson.

Tableau IV. Comparaison des classements des résultats Cobra par rapport aux classements des résultats Référence avec et sans transformation des données Cobra par l'équation de régression.

Comparison of the classification obtained with Cobra results and with the reference results with and without use of the regression equation.

Mesures directes (sans régression)

(\%)
Mesures transformées (avec régression)

(\%)

\begin{tabular}{lrr} 
Identique & 75,2 & 75,6 \\
Meilleur & 8,5 & 17,1 \\
Inférieur & 16,3 & 7,3 \\
\hline
\end{tabular}




\section{Le carry-over effect}

La seringue automatique prélève $100 \mu \mathrm{l}$ de lait et les chasse dans le puits par $800 \mu$ l de solvant triton-trypsine. La conception de l'opération, notamment le rapport égal à 8 entre la quantité de solvant et la quantité de lait poussée dans le puits par le solvant, est telle qu'une contamination résiduelle est improbable. Ce point a été vérifié par le prélèvement séquentiel d'eau stérile et d'échantillons contenant de l'ordre de $10^{6}$ germes $/ \mathrm{ml}$. Le résultat obtenu pour les échantillons d'eau stérile a toujours été $<1000$ germes $/ \mathrm{ml}$, seuil de détermination fixé au niveau de la lecture. Le carry-over effect est donc $<1 / 1000$.

\section{L'influence de la teneur en cellules somatiques}

L'équation de régression multilinéaire faisant intervenir le nombre de cellules somatiques (de moyenne égale à $378000 \mathrm{cel}-$ lules $/ \mathrm{ml}$ avec une amplitude de 25000 à 3216000 cellules $/ \mathrm{ml}$ ) a été établie comme suit, la méthode de référence étant dans ce cas, la méthode Thompson :

$$
\log (\text { Thomp })=0,738+0,470 \log \text { (Cobra) }
$$$$
+0,093 \log \text { (Cellules) avec un } s_{y . x} \text { de } 0,31
$$

La nullité du coefficient affectant log (Cellules) a été testée. Le coefficient n'est pas nul $(P=0,0357<0,05)$. D'un point de vue statistique, l'influence du nombre de cellules somatiques sur le nombre de germes est significative au niveau $\alpha=$ 0,05 . D'un point de vue pratique, cependant, le classement réalisé en tenant compte d'un facteur log (Cellules) dans les trois catégories de germes limitées par les seuils de 100000 et $300000 / \mathrm{ml}$ donne $80,5 \%$ de résultats bien classés au lieu de $79,5 \%$ en négligeant ce facteur.
L'influence du nombre de cellules somatiques sur la détermination du nombre de germes peut en pratique être négligée lorsque cette dernière est effectuée par la technique Cobra.

\section{DISCUSSION ET CONCLUSIONS}

L'évaluation du Cobra 2024 L pour la détermination du nombre de germes dans le lait cru selon la méthode de référence par dilutions a été effectuée dans un laboratoire interprofessionnel belge sans recherche spéciale de performances et avec des laits froids. Le Cobra est probablement la technique automatisée actuelle dont le principe se rapproche le plus de celui de la méthode par dilutions. II fonctionne directement en UFC/ml. Sa répétabilité $(0,238)$ et son GRSD $(21,34 \%)$ sont de l'ordre de ceux des autres méthodes fonctionnant en UFC. Le Cobra ne dissocie pas les amas de bactéries. Cependant, en amont du Cobra au moment du pipettage, on peut avoir une dissociation plus ou moins forte et aléatoire de l'échantillon. Les méthodes par dilutions (Référence) et Thompson comptent aussi des amas, mais la première les dissocie davantage que la seconde.

L'application du Cobra 2024 L en routine a permis une estimation du nombre de germes et un classement des laits crus des producteurs en 3 catégories $(<100000$, 100000 à 300000 et $>300000$ germes/ $\mathrm{ml}$ ) selon la méthode par dilutions. La correspondance atteint $75,2 \%$ de classements identiques dans les mêmes catégories sans intervention d'un calibrage ( $75,6 \%$ avec calibrage). Le Cobra est réglé pour donner une estimation directe des résultats de la méthode de référence. D'autre part, I'utilisation de la méthode Thompson comme méthode de référence rend nécessaire l'intervention d'un cali- 
brage et permet d'atteindre $88,8 \%$ de classements corrects.

Les résultats sont comparables à ceux d'essais effectués par des laboratoires de routine français (LIAL Auch, LIAL Aurillac, Laboratoire régional de contrôle du lait Ceyseriat, 1991) qui ont utilisé la méthode Thompson comme méthode de référence (78 à $82 \%$ de correspondance pour les classements).

La conception et le fonctionnement du système de prélèvement et de distribution des échantillons du Cobra sont tels que le pourcentage de contamination d'un échantillon sur le suivant est $<0,1 \%$.

Le nombre de cellules somatiques exerce une influence statistiquement significative sur la numération bactérienne, mais cette influence est négligeable sur le classement des laits dans les 3 catégories précitées (augmentation de $1 \%$ des échantillons bien classés en tenant compte de la régression). Elle pourrait sans doute être encore réduite en augmentant légèrement la durée de l'action enzymatique lors de la préparation de l'échantillon.

L'appareil modulaire de conception simple installé à Soignies a permis de réaliser des cadences d'analyse élevées (144 échantillons $/ \mathrm{h}$ ). Le réglage a été effectué en fonction d'un compromis imposé entre la précision demandée à différents niveaux et la cadence.

Le Cobra estimant directement et avec une bonne correspondance le nombre de germes dans le lait cru, tel qu'il serait obtenu par la méthode de référence, a donc pu être agréé en Belgique (après la France) comme méthode de routine pour cette détermination dans le cadre du paiement du lait cru au producteur selon la qualité bactériologique.

\section{REMERCIEMENTS}

Les auteurs remercient la société Biocom (Les Ulis Cedex, France) et le Comité interprofessionnel de Soignies (Belgique) pour la mise à disposition de l'appareil, et les nombreuses explications fournies pour l'utilisation du Cobra. Le Comité interprofessionnel de Soignies nous a également fourni les échantillons de lait et le matériel nécessaire à cette étude.

Notre gratitude va également à $\mathrm{R}$ Oger (Centre de recherches agronomiques de Gembloux, Bureau d'informatique et de statistique appliquées) pour l'aide apportée dans l'interprétation des résultats.

\section{RÉFÉRENCES}

Bisconte JC (1990) L'analyse cellulaire et bactérienne en temps réel: le système Cobra. Prog Tech Ing Sci Fr 1, 14-16

Bisconte JC (1991) Face à face avec le Cobra. Rev Lait Fr 504, 60-61

Dasen A, Olid RM, Piton C, Grappin R (1991) Évaluation du Bactoscan 8000 pour la numération automatique et rapide de la flore microbienne du lait cru. Lait 71, 661-670

FIL (1985) Lait. Définition et évaluation de la précision globale des méthodes indirectes d'analyse du lait. Application au calibrage et au contrôle de qualité. Norme provisoire 128 , Fédération internationale de laiterie, Bruxelles

FIL (1991) Lait et produits laitiers. Dénombrement des micro-organismes. Comptage des colonies à $30^{\circ} \mathrm{C}$. Norme $100 \mathrm{~B}$ Fédération Internationale de Laiterie, Bruxelles

Grappin R (1976) Guide pour l'évaluation des méthodes d'analyses de routine. Lait 56 , 608-620

Grappin R (1990) Rapport de la Commission scientifique et technique. Ministère de I'Agriculture. France

Grappin R, Dasen A, Favennec P (1985) Numération automatique et rapide des bactéries du lait cru à l'aide du Bactoscan. Lait 65, 123147 
Pettipher GL, Mansell R, McKinnon CH, Cousins M (1980) Rapid membrane filtration epifluorescent technique for direct enumeration of bacteria in raw milk. Appl Environ Microbiol 39, 423-429

Pettipher GL, Watts YB, Langford SA, Kroll RG (1992) Preliminary evaluation of Cobra an automated DEFT instrument for the Rapid enumeration of microorganisms in cultures, raw milk, meat and fish. Lett Appl Microbiol 14, 206-209
Piton C, Grappin R (1991) A model for statistical evaluation of precision parameters methods : application to dry rehydratable film methods and IDF methods for enumeration of total aerobic mesophilic flora and coliforms en raw milk. J Assoc Off Anal Chem 74, 92-103

Suhren G, Reichmuth J, Heeschen W (1992) Beurteilung von Routinemethoden zur Bestimmung der Bacteriologischen Qualität von Rohmilch. Lebensmittelind Milchwirtsch 1-2, 8-10 